\title{
Influência de Doses Reduzidas do Glyphosate no Tomateiro (Lycopersicon esculentum $)^{1}$
}

\author{
Influence of Reduced Glyphosate Rates on Tomato Plant (Lycopersicon esculentum Mill.)
}

\author{
FIGUEREDO, S.S. ${ }^{2}$, LOECK, A.E. ${ }^{3}$, ROSENTHAL, M.D. ${ }^{4}$, AGOSTINETTO, D. ${ }^{3}$, FONTANA, L.C. ${ }^{2}$ e \\ RIGOLI, R.P. ${ }^{5}$
}

\begin{abstract}
RESUMO - O tomateiro (Lycopersicon esculentum) é cultivado em todas as regiões do Brasil devido à ampla utilização na culinária, tanto in natura quanto industrializado. A deriva do glyphosate vem sendo citada como um dos fatores que interferem negativamente sobre as culturas hortícolas, cultivadas nas adjacências das lavouras de arroz. Objetivou-se com esse verificar os efeitos de doses reduzidas do glyphosate sobre a cultura do tomateiro. $\mathrm{O}$ glyphosate foi aplicado a 0, 15, 30, 45, 60, 75 e 90\% da dose de $1.260 \mathrm{~g}^{-1}$, aos $30,50 \mathrm{e}$ 70 dias após o transplante (DAT) da cultura. As variáveis analisadas foram fitotoxicidade, altura e número de cachos florais, flores e frutos por planta. Os dados experimentais foram submetidos à análise da variância e, sendo significativos, os efeitos de época de aplicação foram comparados pelo teste de Tukey, e os efeitos do fator dose, analisados por regressão. A aplicação do glyphosate, a partir de $378 \mathrm{~g} \mathrm{ha}^{-1}$, afetou o desenvolvimento do tomateiro, independentemente do estádio de desenvolvimento.
\end{abstract}

Palavras-chave: agrotóxicos, deriva, estádio de desenvolvimento, fitotoxicidade, hortaliças.

ABSTRACT - Tomato plant (Lycopersicon esculentum) is grown in all regions in Brazil since it is widely used in cooking, processed or fresh. Glyphosate drift has been found rcited as one of the factors interfering negatively in vegetables cultivated in areas adjacent to rice plantations. The objective of this study was to evaluate the effects of reduced rates of glyphosate on tomato plants, applied at of 0, 15, 30, 45, 60, 75 and 90\% of the commercialdose (1.260 $\left.\mathrm{g} \mathrm{ha}^{1}\right) 30,50$ and 70 days after transplanting (DAT) the tomato plants. The variables analyzed were: phytotoxicity, plant height, number of flowering clusters, and flower and fruits/plant. The data were submitted to analysis of variance, the Tukey test and regression. Glyphosate application at rates higher than $378 \mathrm{~g} \mathrm{ha}^{-1}$ affected the growth of the tomato plants negatively, regardless of their development stage.

Keywords: pesticides, drift, development stage, phytotoxicity, vegetables.

\section{INTRODUÇÃO}

Devido à crescente demanda por maior produção de alimentos, surge a necessidade de maior tecnificação das lavouras para aumentar a produtividade nas áreas agricultáveis.
$\mathrm{Na}$ busca de maior eficiência produtiva, o uso dos agrotóxicos, mais precisamente herbicidas, vem sendo uma ferramenta fundamental.

O tomate (Lycopersicon esculentum) é cultivado em várias regiões do Brasil, sendo

Recebido para publicação em 2.4.2007 e na forma revisada em 29.11.2007.

2 Eng ${ }^{a}-A g r$ à , aluna do Programa de Pós-Graduação em Fitossanidade da Universidade Federal de Pelotas, Caixa Postal 354, 96010900 Capão do Leão-RS, <silmest@ibest.com.br>; ${ }^{3}$ Eng $^{\circ}-$-Agro - Dr., Professor da Faculdade de Agronomia Eliseu Maciel da UFPel, bolsista do CNPq, ${ }^{4}$ Eng $^{\mathrm{a}}$-Agr ${ }^{\mathrm{a}}$, Dra , da Faculdade de Agronomia Eliseu Maciel da UFPel, ${ }^{5}$ Bióloga, aluna do Programa de PósGraduação em Fitossanidade da Universidade Federal de Pelotas. 
utilizado na alimentação humana, tanto in natura como industrializado (Alvarenga, 2000; Filgueira, 2003; Fontes \& Silva, 2005). A área cultivada com tomate no Brasil, no ano de 2005, foi de cerca de 60 mil ha, e o Rio Grande do Sul (RS) participou com 2,4 mil ha, obtendo produção de aproximadamente 107 mil t e produtividade média de $4,4 \mathrm{t} \mathrm{ha}^{-1}$ (IBGE, 2006). Trata-se de uma cultura de elevado risco econômico, em que descuidos em seus tratos culturais podem ocasionar prejuízos irreversiveis.

O arroz irrigado é uma das culturas mais importantes do Estado do Rio Grande do Sul (RS), com produção aproximada de 6,23 milhões de toneladas, em área cultivada de aproximadamente 1,03 milhão de ha e com produtividade média de $6 \mathrm{t}$ ha ${ }^{-1}$ (Gomes \& Magalhães Jr., 2004). Como qualquer cultura agrícola, necessita de bom manejo de plantas daninhas para que possa expressar todo o seu potencial produtivo, o qual geralmente é realizado pela utilização de herbicidas. No entanto, quando da aplicação desses produtos nas adjacências de cultivo de hortaliças, pode ocorrer o fenômeno da deriva.

A deriva pode ocorrer devido ao arraste da calda pulverizada pelo vento ou pela volatilização do produto, sendo dependente das características do herbicida. Outros fatores que podem ser citados, como influentes para ocorrência da deriva, é o tipo de equipamento, calibração, tipo de pontas de pulverização, técnicas de aplicação, condições climáticas e habilidade do operador (Ozkan, 2000; Cunha et al., 2003; Costa et al., 2007; Viana et al. 2007).

O glyphosate é utilizado no Brasil em praticamente todas as culturas agricolas e aplicado em pós-emergência das plantas daninhas. Na cultura do arroz irrigado é utilizado na dessecação de pré-semeadura, no sistema de cultivo mínimo. O glyphosate pertence ao grupo químico das glicinas substituídas, sendo classificado como não-seletivo e de ação sistêmica (Trezzi et al., 2001; Rodrigues \& Almeida, 2005).

A produção das culturas pode ser afetada tanto qualitativa quanto quantitativamente, sem que sintomas visiveis sejam observados em plantas expostas a subdoses de herbicidas.
Nesse sentido, Gilreath et al. (2000) observaram interação significativa entre doses de glyphosate e estádios de desenvolvimento da cultura do pimentão. Os efeitos negativos de doses reduzidas do glyphosate também foram observados nas culturas de milho, sorgo, algodão e eucalipto, em que a intoxicação das plantas resultou em redução de crescimento e produtividade (Alves et al., 2000; Magalhães et al., 2001a, b; Yamashita \& Guimarães, 2005; Tuffi Santos et al., 2006; Yamashita \& Guimarães, 2006).

Os efeitos da deriva simulada do bispyribac-sodium sobre culturas como soja, algodão, sorgo, milho e tomate demonstraram que o tomateiro e o algodoeiro apresentaram maior sensibilidade ao herbicida, cujos sintomas foram clorose nas folhas, retardamento no desenvolvimento e morte do ápice da haste principal (Dillon et al., 2000). Quanto à cultura da beterraba, em diferentes estádios de desenvolvimento, doses reduzidas de dicamba, 2,4$\mathrm{D}$ e picloram provocaram fitotoxicidade, o que resultou em redução na produtividade e na qualidade do produto colhido (Schroeder et al., 1983).

A ocorrência de deriva do glyphosate utilizado na lavoura de arroz irrigado poderá causar injúrias e reduzir a produtividade da cultura do tomateiro. Assim, o objetivo deste trabalho foi verificar os efeitos de doses reduzidas do glyphosate em três estádios de desenvolvimento da cultura do tomateiro.

\section{MATERIAL E MÉTODOS}

O experimento foi realizado em casa de vegetação pertencente à Universidade Federal de Pelotas, localizada no município do Capão do Leão, Rio Grande do Sul.

As mudas foram produzidas em bandejas alveoladas de poliestireno expandido, utilizando sementes do cultivar Kada Gigante, Grupo Santa Cruz, de crescimento indeterminado, sob substrato comercial. O transplante foi realizado quando as mudas estavam no estádio de três folhas definitivas, após 40 dias da semeadura. Em cada recipiente plástico com capacidade volumétrica de $7 \mathrm{~L}$ foram transplantadas duas mudas; após uma semana, realizou-se o desbaste, permanecendo uma 
planta por recipiente, as quais constituíram as unidades experimentais. Estas foram distanciadas de $1 \mathrm{~m}$, para evitar o contato entre as folhas de plantas de diferentes tratamentos.

A adubação foi realizada conforme a análise química do substrato, o qual foi mantido com o teor de umidade próximo da capacidade máxima de retenção, durante toda a fase experimental. Foram realizados também os tratos culturais necessários à boa manutenção das plantas, com retirada de ramos axilares das folhas (desbrota) e das estruturas vegetativas do prolongamento dos cachos florais e folhas portadoras de injúrias fisicas ou senescentes.

O delineamento experimental adotado foi o de blocos ao acaso, com quatro repetições. Os tratamentos foram arranjados em esquema fatorial, em que o fator A testou épocas de aplicação do herbicida em fases de desenvolvimento do tomateiro aos 30 dias após o transplante (DAT) (pleno período vegetativo), 50 DAT (presença uniforme dos primeiros cachos florais, caracterizando o início do período reprodutivo) e 70 DAT (presença de frutos com diâmetro médio aproximado de $2,5 \mathrm{~cm}$ ), que corresponderam respectivamente a plantas com $5-8,10-12$ e $14-16$ folhas, e o fator B avaliou doses do glyphosate de $0,15,30,45,60$, 75 e $90 \%$ da dose comercial de $1.260 \mathrm{~g} \mathrm{ha}^{-1} \mathrm{de}$ glyphosate.

As aplicações foram feitas com o auxílio de pulverizador costal pressurizado a $\mathrm{CO}_{2}$ a $0,4 \mathrm{~m}$ acima do topo da planta, com ponta tipo leque 110.015 e pressão de trabalho de $210 \mathrm{kPa}$, calibrado para um volume de calda de $150 \mathrm{~L} \mathrm{ha}^{-1}$.

As variáveis respostas foram avaliadas semanalmente, até 28 dias após aplicação (DAA). Para determinação de fitotoxicidade utilizou-se uma escala de zero (0) a cem (100), em que zero representa ausência de sintomas e 100 corresponde à morte da planta. A altura das plantas foi avaliada com o auxílio de uma régua graduada, iniciando a medição da base da planta ao ápice. O número de cachos florais, flores e frutos foi avaliado pela contagem nas fases de desenvolvimento de 50 e 70 DAT.

Os dados foram analisados quanto à sua homocedasticidade e posteriormente submetidos à análise de variância $(\mathrm{p}<0,05)$. Os efeitos de épocas de aplicação foram comparados pelo teste de Tukey $(p<0,05)$, e os efeitos do fator dose, avaliados por modelos de regressão linear e não-linear $(\mathrm{p}<0,05)$.

\section{RESULTADOS E DISCUSSÃO}

Para a variável fitotoxicidade, houve interação entre época de aplicação e doses do herbicida (Tabela 1). Na avaliação realizada aos 7 DAA, não houve diferença na fitotoxicidade entre as épocas de aplicação, à exceção das doses de 30 e $90 \%$, em que os efeitos tóxicos aos 30 DAT foram superiores aos observados na aplicação aos 70 DAT. Nas demais avaliações de fitotoxicidade não houve diferença entre as épocas de aplicação, à exceção de $15 \mathrm{e}$ $30 \%$ da dose utilizada. Quanto à dose de 15\%, a maior fitotoxicidade foi verificada quando a aplicação foi feita aos 70 DAT. Já para a dose de $30 \%$, em geral, a maior fitotoxicidade foi observada na primeira época de aplicação.

De modo geral, as plantas de tomate, em todas as épocas de avaliação, mostraram menor fitotoxicidade quando a aplicação de doses reduzidas de glyphosate foi feita aos 70 DAT (Tabela 1). Isso se deve ao fato de as plantas em estádios iniciais de desenvolvimento apresentarem maior sensibilidade ao herbicida, quando comparadas às plantas mais desenvolvidas, como foi observado também por Gilreath et al. (2000).

A resposta da fitotoxicidade do glyphosate ao tomateiro ajustou-se à equação exponencial em todas as épocas de aplicação ou avaliação (Figura 1). Os valores do coeficiente de determinação $\left(R^{2}\right)$ variaram de 0,80 a 0,99 , demonstrando haver bom ajuste dos dados ao modelo (Tabela 2).

A aplicação das doses a partir de $378 \mathrm{~g} \mathrm{ha}^{-1}$ $(30 \%)$ nas três fases de desenvolvimento gerou sintomas que caracterizam a ação fitotóxica do glyphosate. A injúria aumentou com o incremento da dose do produto em todos os períodos de avaliação; a partir dos 14 DAA, são evidenciados sintomas de fitotoxicidade próxima de $100 \%$. Inicialmente observou-se paralisação no crescimento do tomateiro, seguida por murcha. Nas avaliações subseqüentes foi constatada clorose, seguida de necrose e morte das plantas, concordando com o quadro 
Tabela 1 - Fitotoxicidade do glyphosate às plantas de tomate, cultivar Kada Gigante, em função de época de aplicação e dose FAEM/UFPel, Capão do Leão-RS, 2005

\begin{tabular}{|c|c|c|c|c|c|c|c|}
\hline \multirow{2}{*}{ 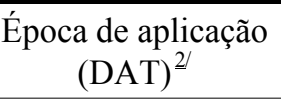 } & \multicolumn{7}{|c|}{ Dose $(\%)^{\underline{1} /}$} \\
\hline & 0 & 15 & 30 & 45 & 60 & 75 & 90 \\
\hline \multicolumn{8}{|c|}{ Fitotoxicidade (\%) $7 \mathrm{DAA}^{3 /}$} \\
\hline 30 & $0,0^{\mathrm{ns}}$ & $1,3 \mathrm{a}$ & $40,0 \mathrm{a}$ & $50,0 \mathrm{a}$ & $48,8 \mathrm{a}$ & $57,5 \mathrm{a}$ & $72,5 \mathrm{a}$ \\
\hline 50 & 0,0 & $0,0 \mathrm{a}$ & $20,0 \mathrm{ab}$ & $37,5 \mathrm{a}$ & $43,8 \mathrm{a}$ & $55,0 \mathrm{a}$ & $66,3 \mathrm{ab}$ \\
\hline 70 & 0,0 & $8,8 \mathrm{a}$ & $18,8 \mathrm{~b}$ & $33,8 \mathrm{a}$ & $41,3 \mathrm{a}$ & $48,8 \mathrm{a}$ & $51,3 \mathrm{~b}$ \\
\hline \multicolumn{8}{|c|}{ Fitotoxicidade (\%) 14 DAA } \\
\hline 30 & 0,0 & $2,5 \mathrm{~b}$ & $71,3 \mathrm{a}$ & $81,3 \mathrm{a}$ & $77,5 \mathrm{a}$ & $93,8 \mathrm{a}$ & $97,5 \mathrm{a}$ \\
\hline 50 & 0,0 & $5,0 \mathrm{~b}$ & $37,5 \mathrm{~b}$ & $60,0 \mathrm{a}$ & $77,5 \mathrm{a}$ & $82,5 \mathrm{a}$ & $85,0 \mathrm{a}$ \\
\hline 70 & 0,0 & $50,0 \mathrm{a}$ & $57,5 \mathrm{ab}$ & $70,0 \mathrm{a}$ & $81,3 \mathrm{a}$ & $86,3 \mathrm{a}$ & $91,3 \mathrm{a}$ \\
\hline \multicolumn{8}{|c|}{ Fitotoxicidade (\%) 21 DAA } \\
\hline 30 & 0,0 & $2,5 \mathrm{~b}$ & $100,0 \mathrm{a}$ & $100,0 \mathrm{a}$ & $95,0 \mathrm{a}$ & $100,0 \mathrm{a}$ & $100,0 \mathrm{a}$ \\
\hline 50 & 0,0 & $5,0 \mathrm{~b}$ & $80,0 \mathrm{~b}$ & $97,5 \mathrm{a}$ & $96,3 \mathrm{a}$ & $97,5 \mathrm{a}$ & $95,0 \mathrm{a}$ \\
\hline 70 & 0,0 & $55,0 \mathrm{a}$ & $70,0 \mathrm{~b}$ & $85,0 \mathrm{a}$ & $88,8 \mathrm{a}$ & $93,3 \mathrm{a}$ & $95,8 \mathrm{a}$ \\
\hline \multicolumn{8}{|c|}{ Fitotoxicidade (\%) 28 DAA } \\
\hline 30 & 0,0 & $0,0 \mathrm{~b}$ & $100,0 \mathrm{a}$ & $100,0 \mathrm{a}$ & $100,0 \mathrm{a}$ & $100,0 \mathrm{a}$ & $100,0 \mathrm{a}$ \\
\hline 50 & 0,0 & $2,5 \mathrm{~b}$ & $88,8 \mathrm{ab}$ & $100,0 \mathrm{a}$ & $97,5 \mathrm{a}$ & $100,0 \mathrm{a}$ & $93,8 \mathrm{a}$ \\
\hline 70 & 0,0 & $60,0 \mathrm{a}$ & $73,8 \mathrm{~b}$ & $82,5 \mathrm{a}$ & $90,0 \mathrm{a}$ & $95,0 \mathrm{a}$ & $96,5 \mathrm{a}$ \\
\hline
\end{tabular}

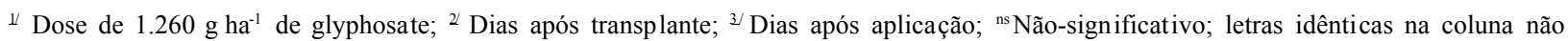
diferem estatisticamente entre épocas pelo teste de Tukey $(\mathrm{p} \leq 0,05)$.
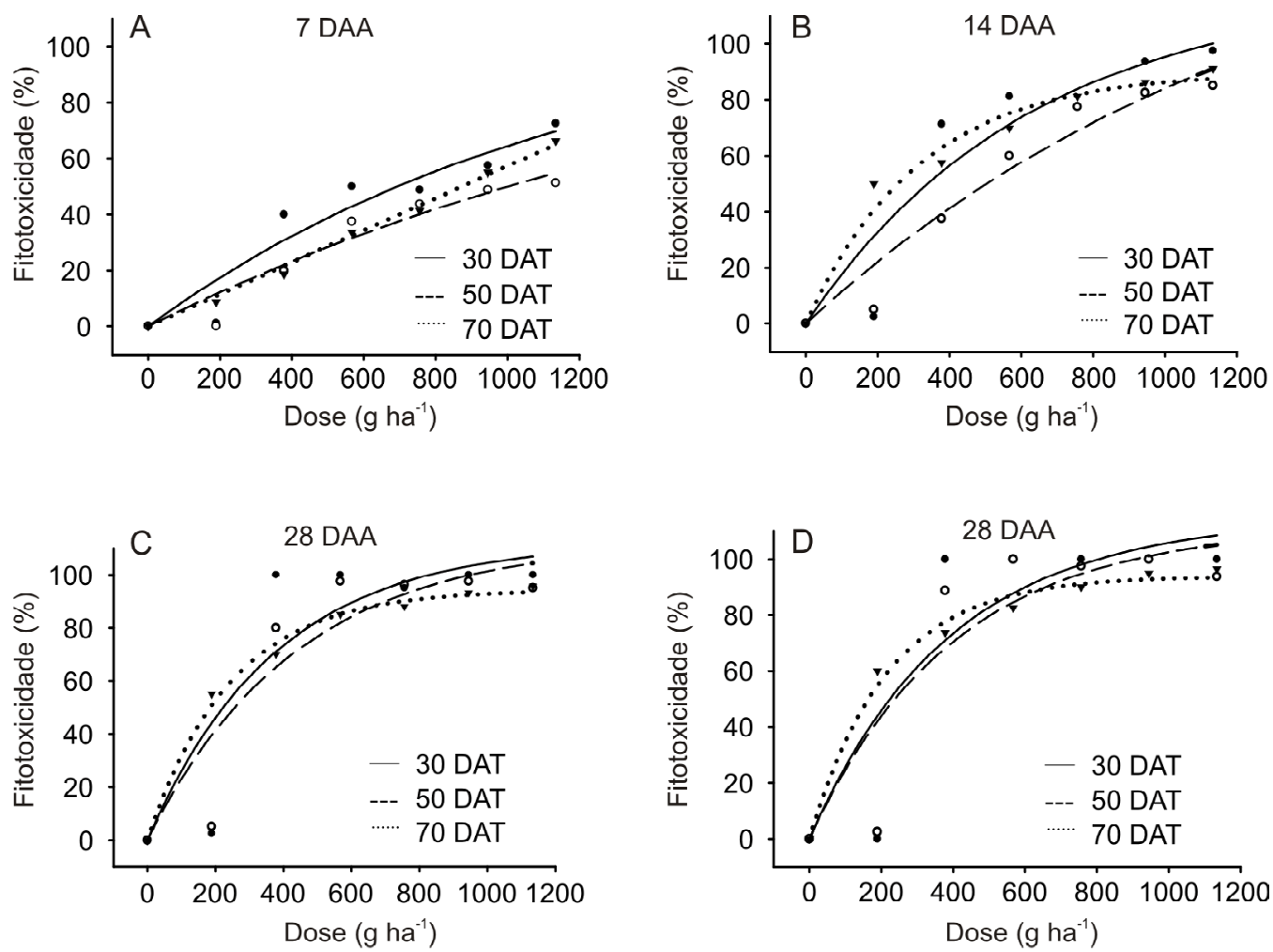

Figura 1 -Fitotoxicidade do herbicida glyphosate à cultura do tomate, cultivar Kada Gigante, nas aplicações realizadas aos $30(\bullet)$, 50 ( ○) e 70 ( $\mathbf{\nabla})$ dias após transplante (DAT) - FAEM/UFPel, Capão do Leão-RS, 2005. 
Tabela 2 - Equações de fitotoxicidade do glyphosate sobre a cultura do tomate, cultivar Kada Gigante - FAEM/UFPel, Capão do Leão-RS, 2005

\begin{tabular}{|c|c|c|c|c|}
\hline Avaliação & Época de aplicação & Equação & $\mathrm{R}^{2}$ & QMR \\
\hline \multirow{3}{*}{$7 \mathrm{DAA}^{1 /}$} & $30 \mathrm{DAT}^{2 /}$ & $Y=114,60\left(1-e^{-0,0008 x}\right)$ & 0,91 & 84,10 \\
\hline & $50 \mathrm{DAT}$ & $\mathrm{Y}=119,77\left(1-\mathrm{e}^{-0,0005 x}\right)$ & 0,93 & 40,23 \\
\hline & $70 \mathrm{DAT}$ & $Y=509547,37\left(1-\mathrm{e}^{-0,0001 x}\right)$ & 0,99 & 4,20 \\
\hline \multirow{3}{*}{14 DAA } & $30 \mathrm{DAT}$ & $\mathrm{Y}=119,63\left(1-\mathrm{e}^{-0,0016 \mathrm{x}}\right)$ & 0,88 & 251,85 \\
\hline & $50 \mathrm{DAT}$ & $Y=160,59\left(1-e^{-0,0007 x}\right)$ & 0,95 & 79,83 \\
\hline & 70 DAT & $Y=90,05\left(1-e^{-0,0032 x}\right)$ & 0,97 & 31,64 \\
\hline \multirow{3}{*}{$21 \mathrm{DAA}$} & $30 \mathrm{DAT}$ & $Y=112,68\left(1-e^{-0,0026 x}\right)$ & 0,80 & 560,17 \\
\hline & $50 \mathrm{DAT}$ & $\mathrm{Y}=113,02\left(1-\mathrm{e}^{-0,0023 \mathrm{x}}\right)$ & 0,86 & 351,90 \\
\hline & $70 \mathrm{DAT}$ & $Y=94,42\left(1-e^{-0,0041 x}\right)$ & 0,99 & 9,21 \\
\hline \multirow{3}{*}{$28 \mathrm{DAA}$} & $30 \mathrm{DAT}$ & $\mathrm{Y}=114,74\left(1-\mathrm{e}^{-0,0026 x}\right)$ & 0,80 & 602,44 \\
\hline & $50 \mathrm{DAT}$ & $\mathrm{Y}=111,76\left(1-\mathrm{e}^{-0,0025 \mathrm{x}}\right)$ & 0,82 & 471,65 \\
\hline & $70 \mathrm{DAT}$ & $Y=93,92\left(1-e^{-0,0046 x}\right)$ & 0,99 & 15,85 \\
\hline
\end{tabular}

${ }^{1 /}$ Dias após a aplicação; ${ }^{2}$ Dias após o transplante.

sintomatológico referente a esse herbicida, relatado por Trezzi et al. (2001).

Esses dados são concordantes com os observados por Russo (1990), cujos sintomas visuais na cultura do tomateiro exposta às doses reduzidas de glyphosate foram clorose em folhas jovens e no ápice da haste principal. $\mathrm{O}$ glyphosate, por ser um herbicida não-seletivo, quando utilizado em doses reduzidas crescentes, promove aumento progressivo na injúria foliar, reduzindo o vigor de plantas e, conseqüentemente, também a produtividade na cultura do pimentão (Gilreath et al., 2000).

Quanto à variável altura de planta, embora tenha havido interação entre os fatores época e doses do glyphosate, esta não foi considerada, visto que a aplicação de doses reduzidas foi realizada em três fases distintas da cultura, sendo incoerente a comparação entre ambas. Os valores do coeficiente de determinação $\left(R^{2}\right)$ variaram de 0,80 a 0,97, demonstrando haver bom ajuste dos dados ao modelo (Tabela 3 ).

Os dados de altura de planta ajustaram-se à equação quadrática; o aumento nas doses, em todas as fases de desenvolvimento, promoveu redução da altura das plantas, com exceção da aplicação aos 30 DAT (Figura 2). Esse comportamento pode ser explicado pelo fato de nesta fase de desenvolvimento a planta se apresentar mais suscetivel à ação do herbicida e, por conseguinte, apresentar menores chances de recuperação. O crescimento das plantas foi maior à medida que se retardou a aplicação do herbicida, com maior altura observada aos 70 DAT.

No tocante à variável número de cachos florais, não houve interação entre os fatores estudados. Já para as variáveis número de flores e frutos, embora tenha havido interação entre os fatores, estas não foram consideradas, visto que a simulação da deriva foi realizada em três fases distintas da cultura, sendo inconsistente a comparação. No tratamento realizado aos 30 DAT, não houve contagem de cachos florais, flores e frutos em razão de a última avaliação ter sido realizada aos 28 DAA, não havendo essas estruturas.

As variáveis número de cachos florais, flores e frutos ajustaram-se adequadamente ao modelo exponencial em todas as épocas de aplicação e de avaliação. Os valores do coeficiente de determinação $\left(\mathrm{R}^{2}\right)$ variaram de 0,78 a 0,99 (Figura 3).

O número de cachos florais por planta foi reduzido com o incremento da dose do glyphosate, em todas as épocas de aplicação. A produção 
Tabela 3 - Equações referentes à altura de plantas de tomate, cultivar Kada Gigante, em função de aplicações de doses do glyphosate - FAEM/UFPel, Capão do Leão-RS, 2005

\begin{tabular}{|c|c|c|c|c|}
\hline Avaliação & Época de aplicação & Equação & $\mathrm{R}^{2}$ & QMR \\
\hline \multirow{3}{*}{$7 \mathrm{DAA}^{1 /}$} & $30 \mathrm{DAT}^{2 /}$ & ns & - & - \\
\hline & 50 DAT & $Y=101,93-0,043 x+0,0001 x^{2}$ & 0,97 & 1,21 \\
\hline & 70 DAT & $Y=148,03-0,068 x+0,0001 x^{2}$ & 0,80 & 40,68 \\
\hline \multirow{3}{*}{14 DAA } & 30 DAT & $Y=82,07-0,04 x+0,0001 x^{2}$ & 0,83 & 28,74 \\
\hline & 50 DAT & $Y=114,25-0,08 x+0,0001 x^{2}$ & 0,95 & 8,79 \\
\hline & 70 DAT & $Y=164,96-0,10 x+0,0001 x^{2}$ & 0,86 & 76,13 \\
\hline \multirow{3}{*}{$21 \mathrm{DAA}$} & $30 \mathrm{DAT}$ & $Y=94,86-0,075 x+0,0001 x^{2}$ & 0,83 & 75,06 \\
\hline & 50 DAT & $Y=121,20-0,099 x+0,0001 x^{2}$ & 0,95 & 13,68 \\
\hline & $70 \mathrm{DAT}$ & $Y=172,54-0,12 x+0,0001 x^{2}$ & 0,89 & 80,32 \\
\hline \multirow{3}{*}{$28 \mathrm{DAA}$} & $30 \mathrm{DAT}$ & $Y=111,52-0,29 x+0,0002 x^{2}$ & 0,85 & 524,26 \\
\hline & 50 DAT & $Y=136,02-0,14 x+0,0001 x^{2}$ & 0,92 & 49,22 \\
\hline & 70 DAT & $Y=181,52-0,14 x+0,0001 x^{2}$ & 0,89 & 109,14 \\
\hline
\end{tabular}

${ }^{1}$ Dias após aplicação; ${ }^{2 /}$ Dias após o transplante.
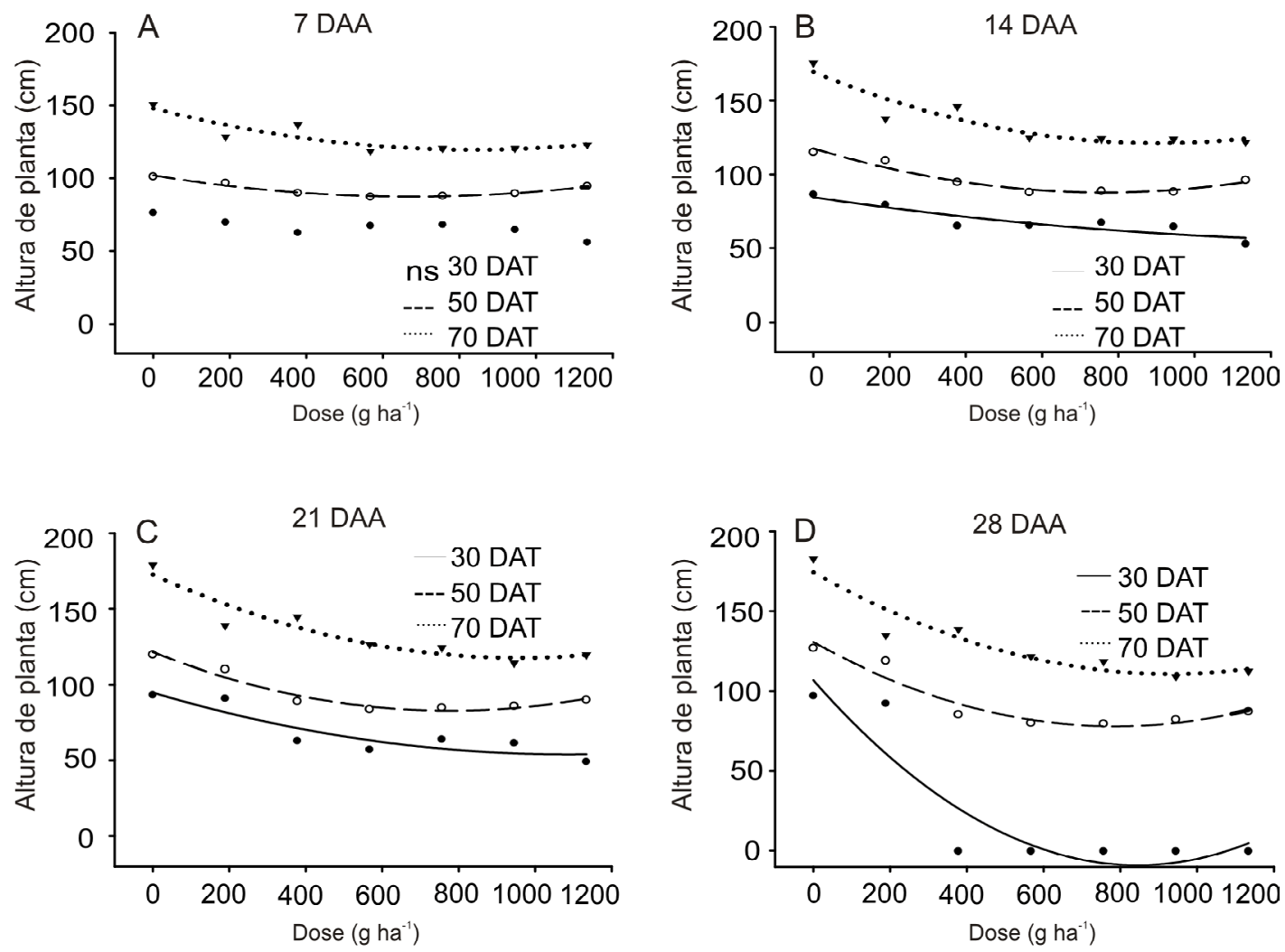

Figura 2 - Altura de plantas de tomate, cultivar Kada Gigante, em função de aplicações de doses do herbicida glyphosate, realizadas aos $30(\bullet), 50$ ( ○) e 70 ( $\boldsymbol{\nabla})$ dias após transplante (DAT). FAEM/UFPel - Capão do Leão-RS, 2005. 

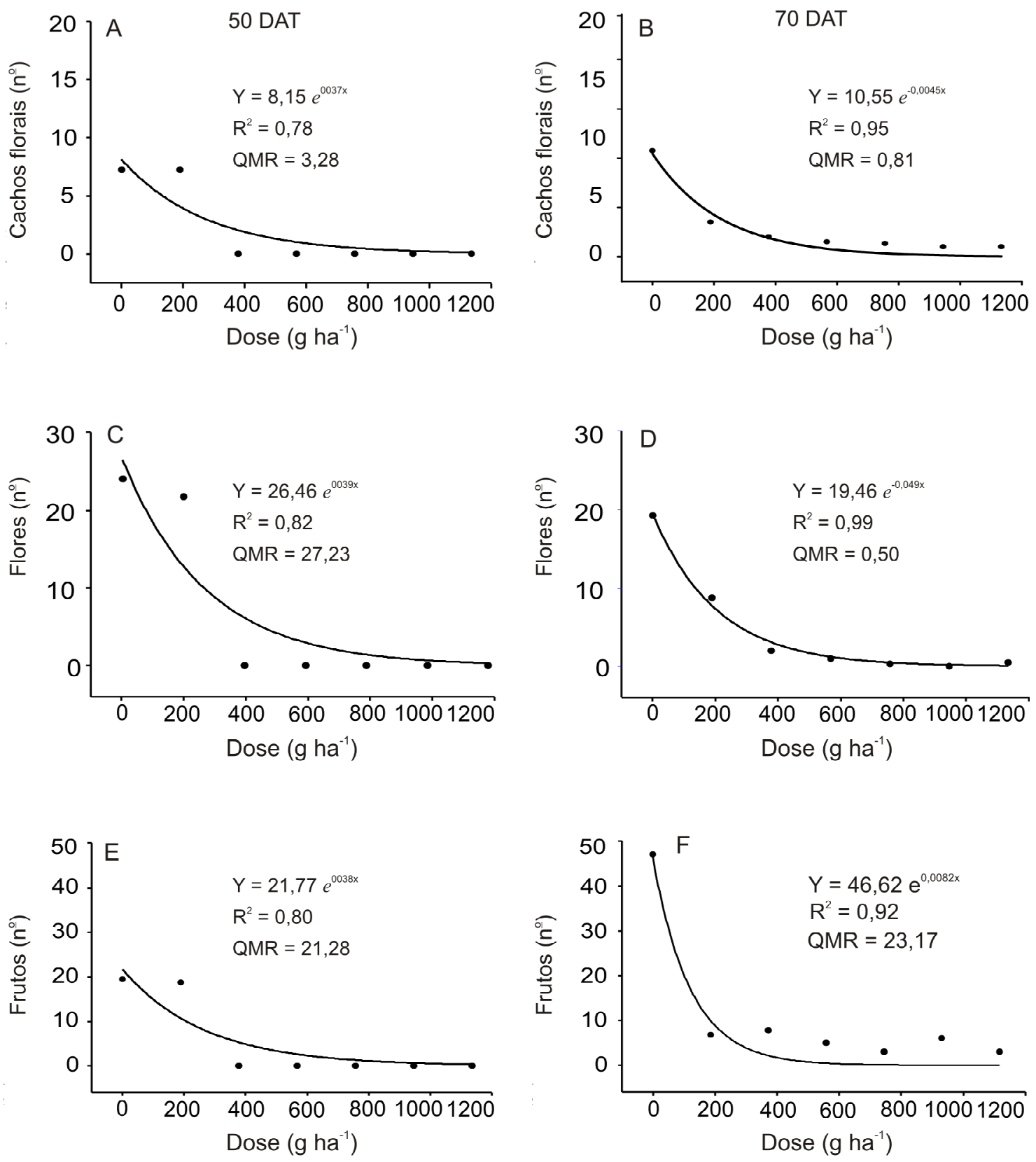

Figura 3 - Número médio de cachos florais, flores e frutos por planta de tomate, cultivar Kada Gigante, em função de doses do herbicida glyphosate, avaliado aos 28 dias após a aplicação - FAEM/UFPel, Capão do Leão-RS, 2005.

de cachos florais aos 70 DAT foi reduzida na dose de $189 \mathrm{~g} \mathrm{ha}^{-1}(15 \%)$, quando comparada aos 50 DAT, podendo isso ser observado pela maior inclinação da curva (parâmetro b da equação), o qual demonstra maior redução para menores doses (Figura 3).

O número de flores por planta foi reduzido com o incremento da dose do glyphosate, em todas as fases de aplicação (Figura 3). Para a mesma variável foi observado número superior na dose de $189 \mathrm{~g} \mathrm{ha}^{-1}(15 \%)$ do glyphosate, com aborto floral nas maiores doses.

O número de frutos por planta foi reduzido com o incremento da dose do herbicida, em todas as fases de aplicação (Figura 3). A produção de frutos foi superior na testemunha 
aos 70 DAT, comparado aos 50 DAT, em função do desenvolvimento da planta. No entanto, a partir da dose de $189 \mathrm{~g} \mathrm{ha}^{-1}(15 \%)$, a redução do número frutos foi superior aos 70 DAT.

Quando da aplicação aos 50 e 70 DAT, observou-se clorose nos cachos florais, com posterior aborto floral. O mesmo comportamento foi obser lo na cultura do tomateiro por Fagliari et al. (2004), os quais verificaram, nas plantas submetidas a subdoses de 2,4-D, necrose e aborto de botões florais, resultando em menor número de frutos por planta; a tolerância do tomateiro aumenta com a idade, especialmente após a frutificação.

A área foliar é importante, por ser a fonte supridora. Logo, sua redução acarreta problemas para a planta durante o período reprodutivo, uma vez que as folhas, juntamente com o colmo, são as principais fontes para suprir, neste período, a alta demanda por fotoassimilados formados através da fotossintese (Magalhães et al., 2001b).

Os resultados obtidos demonstram que a aplicação de doses reduzidas do glyphosate interfere negativamente na cultura do tomateiro. A dose de $189 \mathrm{~g} \mathrm{ha}^{-1}$ ocasionou fitotoxicidade quando aplicada aos 70 dias após o transplante. A aplicação de doses a partir de $378 \mathrm{~g} \mathrm{ha}^{-1}$ aumenta a fitotoxicidade e reduz a altura de planta, o número de cachos, flores e frutos de tomate, independentemente do estádio de desenvolvimento da cultura.

\section{LITERATURA CITADA}

ALVES, L. W. R. et al. Efeito da aplicação de subdoses dos herbicidas glyphosate e oxyfluorfen, simulando deriva sobre a cultura do milho (Zea mays L.). Ci. Agrotec., v. 24, p. $889-897,2000$.

ALVARENGA, M. A. R. Cultura do tomateiro. Lavras: Universidade Federal de Lavras, 2000. 91 p.

COSTA, A. G. F. et al. Efeito da intensidade do vento, da pressão e de pontas de pulverização na deriva de aplicações de herbicidas em pré-emergência. Planta Daninha, v. 25, n. 1, p. 203-210, 2007.

CUNHA, J. P. A. R. et al. Avaliação de estratégias para redução da deriva de agrotóxicos em pulverizações hidráulicas. Planta Daninha, v. 21, n. 2, p. 325-332, 2003.
DILLON, T. L. et al. Response of selected crops to simulated drift of bispyribac-sodium, 2000. Disponível em: $<$ http://www.swss.ws/Proceedings/pdf $>$ Acesso em: 7 de fev. de 2006.

FAGLIARI, J. R. et al. Efeitos e impacto econômico da aplicação de subdoses de 2,4-D, simulando deriva, sobre o tomateiro (Lycopersicon esculentum). In: CONGRESSO BRASILEIRO DA CIÊNCIA DAS PLANTAS

DANINHAS, 24., 2004, São Pedro. Resumos... São Pedro: 2004. p. 200.

FILGUEIRA, F. A. R. Solanáceas. Agrotecnologia moderna na produção de tomate, batata, pimentão, pimenta, berinjela e jiló. Lavras: Universidade Federal de Lavras, 2003. 333 p.

FONTES, P. C. R.; SILVA, D. J. H. Olericultura: teoria e prática. Viçosa, MG: Universidade Federal de Viçosa, 2005. $486 \mathrm{p}$.

FRANS, R.; TALBERT, R.; MARX, D. Experimental design and techniques for measuring and analyzing plant responses to weed control practices. In: CARPER, N. D. Research methods in weed science. 3.ed. Champaing: Southern Weed Science Soci 1986, p. 37.

GILREATH, J. P.; CHASE, C. A.; LOCASCIO, S. J. Phytotoxic effects of glyphosate on pepper (Capsicum annuиm). Weed Technol., v. 14, p. 488-494, 2000.

GOMES, A. S.; MAGAlHÃES JR., A. M. Arroz irrigado no Sul do Brasil. Brasília: EMBRAPA Pelotas Informação Tecnológica, 2004. 899 p.

INSTITUTO BRASILEIRO DE GEOGRAFIA E ESTATÍSTICA - IBGE. Disponível em: <http:// www.sidra.ibge.gov.br> Acesso em: 7 de fev. de 2006.

MAGALHÃES, P. C. et al. Efeito de doses reduzidas de glyphosate e paraquat simulando deriva na cultura do milho. Planta Daninha, v. 19, n. 2, p. 247-253, 2001 a.

MAGALHÃES, P. C. et al. Efeito de doses reduzidas de glyphosate e paraquat simulando deriva na cultura do sorgo. Planta Daninha, v. 19, p. 255-262, 2001b.

OZKAN, H. E. Reducing spray drift, 2000. Disponível em: $<$ http://ohioline.osu. edu/b816/index.html $>$ Acesso em: 25 de fev. de 2007.

RODRIGUES, B. N.; ALMEIDA, F. S. Guia de herbicidas. 5.ed. Londrina: Grafmarke, 2005. p. 275-289.

RUSSO, V. M. Reaction of tomato cultivars to a sublethal dose of glyphosate. Hortsci., v. 25, p. 162-166, 1990. 
SCHROEDER, G. L.; COLE, D. F.; DEXTER, A. G. Sugarbeet (Beta vulgaris L.) response to simulated herbicide spray drift. Weed Sci., v. 31, p. 831-836, 1983.

TREZZI, M. M.; KRUSE, N. D.; VIDAL, R. A. Inibidores de EPSPS. In: VIDAL, R. A.; MEROTTO JR., A.

Herbicidologia. Porto Alegre: Evangraf, 2001. p. 37-45.

TUFFI SANTOS, L. D. et al. Intoxicação de espécies de eucalipto submetidas à deriva do glyphosate. Planta

Daninha, v. 24, n. 2, p. 359-364, 2006.
VIANA, R. G. et al. Características técnicas de pontas de pulverização LA-1JC e SR-1. Planta Daninha, v. 25, n. 1, p. 211-218, 2007.

YAMASHITA, O. M.; GUIMARÃES, S. C. Deriva simulada de glyphosate em algodoeiro: efeito de dose, cultivar e estádio de desenvolvimento. Planta Daninha, v. 24, n. 4, p. 821-826, 2006.

YAMASHITA, O. M.; GUIMARÃES, S. C. Resposta de cultivares de algodoeiro a subdoses de glyphosate. Planta Daninha, v. 23, n. 4, p. 627-633, 2005. 